This item was submitted to Loughborough's Research Repository by the author.

Items in Figshare are protected by copyright, with all rights reserved, unless otherwise indicated.

\title{
Experimental investigation of helicity in turbulent swirling jet using dual-plane dye laser PIV technique
}

\author{
PLEASE CITE THE PUBLISHED VERSION
}

http://dx.doi.org/10.1007/s00348-008-0515-3

\section{PUBLISHER}

(C) Springer

\section{VERSION}

NA (Not Applicable or Unknown)

\section{PUBLISHER STATEMENT}

This work is made available according to the conditions of the Creative Commons Attribution-NonCommercialNoDerivatives 4.0 International (CC BY-NC-ND 4.0) licence. Full details of this licence are available at: https://creativecommons.org/licenses/by-nc-nd/4.0/

\section{LICENCE}

CC BY-NC-ND 4.0

\section{REPOSITORY RECORD}

Regunath, Gavita S., William B. Zimmerman, Vaclav Tesar, and Buddhika N. Hewakandamby. 2017. "Experimental Investigation of Helicity in Turbulent Swirling Jet Using Dual-plane Dye Laser PIV Technique". figshare. https://hdl.handle.net/2134/24273. 


\title{
Experimental investigation of helicity in turbulent swirling jet using dual-plane dye laser PIV technique
}

\author{
Gavita S. Regunath · William B. Zimmerman • \\ Václav Tesař · Buddhika N. Hewakandamby
}

Received: 13 December 2006/Revised: 6 May 2008/ Accepted: 7 May 2008/Published online: 10 July 2008

(C) Springer-Verlag 2008

\begin{abstract}
This paper reports a new method of generating two light sheets using a dye laser system and the use of this dual-plane dye laser system to analyse average helicity and energy dissipation in a turbulent swirling flow. The dualplane PIV system that was used in this study consisted of three cameras and a single frequency Nd:YAG laser, which was used to generate two parallel light sheet planes with differing wavelengths(colour). The method of generating two different light sheet wavelengths using a single laser source is an innovative and new technique. Stereoscopic PIV measurements were obtained in one plane with the use of two CCD cameras, and standard PIV measurements were obtained in the other plane with the use of one CCD camera. The light scattered by the particles on two different light sheets were separated using appropriate optical filters. The measurements obtained were used to estimate the components of the velocity gradient tensor. The tensor components were then used to determine the average vorticity components and helicity quantities of the fluid that was investigated. To determine the average turbulent kinetic energy dissipation, the continuity equation was used to infer the out-of-plane gradient of the out-of-plane velocity. From the analysis of the results, it was found that
\end{abstract}

G. S. Regunath $(\bowtie) \cdot$ W. B. Zimmerman ·

B. N. Hewakandamby

Department of Chemical and Process Engineering,

University of Sheffield, Newcastle Street,

Sheffield S1 3JD, UK

e-mail: G.S.Regunath@lboro.ac.uk

V. Tesař

Institute of Thermomechanics,

Academy of Sciences of the Czech Republic,

Narodni 3, Czech Republic regions with high helicity were correlated with regions of high turbulent kinetic energy dissipation.

\section{Introduction}

Particle image velocimetry (PIV) is a measurement technique for obtaining instantaneous whole field velocities. It is a non-intrusive, whole field optical measuring technique used to obtain velocity information about fluid motion. It is well known that by analysing the velocity gradients, more information such as vorticity, energy dissipation, helicity, Reynolds stresses, etc, can be determined. These quantities can help elucidate fluid mechanical features intrinsic to the flow. However, estimations of the velocity gradient tensor is a challenging task which requires acquisition of all the three components of the velocity vector at a number of points that are slightly displaced along three mutually perpendicular directions.

With the 2D PIV technique, which uses a single two dimensional light sheet to provide two components of velocity vectors $u$ and $v$, only four out of the nine velocity gradient components $\partial u_{i} / \partial x_{j}$ can be computed. Consequently, with relation to computing the vorticity vector for a given fluid flow, only the $\omega_{z}$ vorticity component can be determined. Stereoscopic particle image velocimetry (3D PIV) on the other hand can yield additional information about the third (out-of-plane) component of velocity, $w$ and therefore can provide two further velocity gradient components. However with regards to the vorticity vector, the $\omega_{z}$ vorticity component can only be determined which is similar to what the 2D PIV can determine as it lacks contributions from out-of-plane derivatives.

With the use of an advanced PIV technique, the dualplane PIV system, all components of the velocity gradient, 
$\partial u_{i} / \partial x_{j}$ can be estimated, and hence all the vorticity components, $\omega_{x}, \omega_{y}$ and $\omega_{z}$ can be determined from the velocity measurements. Unlike the standard 2D PIV and the stereoscopic PIV technique, the dual-plane PIV technique can provide more information to help understand and analyse the physics of flow. The dual-plane PIV was first developed by Kähler and Kompenhans (1999) where two spatially separated light sheets were used to measure instantaneous velocities in fluid flow.

Over recent years, researchers have used the dual-plane PIV method to analyse all nine velocity gradients simultaneously. Kähler and Kompenhans (1999), Kähler (2004) used the polarisation based dual-plane PIV technique with four cameras to investigate turbulent structures in turbulent boundary layers. Hu et al (2002) used the similar dual-plane PIV setup to measure the air jet flow, which was exhausted from a lobbed nozzle. They successfully visualised the evolution and interaction of large-scale vortices in the lobbed jet mixing flow at different streamwise distances.

Mullin and Dahm (2005) reported a new concept of dual-plane PIV system that used two different light sheet wavelengths, optical filters and four cameras (two-frequency dual-plane PIV). This technique allowed the measurements of all nine components of the velocity gradients, $\partial u_{i} / \partial x_{j}$ at the intermediate and small scales of a turbulent shear flow. Ganapathisubramani et al (2005) used the polarisation based dual-plane PIV to measure a turbulent boundary layer. However, instead of using the four camera setup, they used three cameras along with the continuity equation to estimate all the nine velocity gradient fields. Kim et al (2006) used the polarisation based dual-plane PIV technique and the three camera setup to measure three-dimensional vortical structures in a turbulent boundary layer flow. However, instead of using two parallel planes, they used orthogonal planes to demonstrate the presence of coherent structures.

The dual-plane PIV technique has been shown to be an invaluable and accurate measurement technique, which can be used to successfully determine the complete velocity gradient components, Saikrishnan et al (2006). In addition, using any of the two reported setups; the four camera setup or the three camera setup, the complete velocity gradient component, $\partial u_{i} / \partial x_{j}$ can be determined. However, for the three camera system the continuity equation for incompressible flows is required to compute the all velocity gradient components.

In the present study, the results of the dual-plane PIV technique that was used to analyse a turbulent swirling jet are presented. The dual-plane PIV technique employed in this study was based on a three camera setup and the use of two different light sheet wavelengths generated in a novel manner. The dual-plane PIV systems that have been used and reported so far are all based on either the polarisation based dual-plane technique or the frequency-based dualplane technique that require two independent laser sources to generate two light sheets. The technique used in this study, the dual-plane dye laser system uses a single laser source. The two different light sheets are produced using a dye laser box.

The use of the novel dual-plane dye laser PIV system will demonstrate the achievements in instantaneously obtaining all three components of the vorticity and velocity fields in a turbulent swirling flow field. The vorticity components obtained will be used to evaluate average helicity and using the continuity equation the average turbulent kinetic energy dissipation rate will also be estimated. To the authors' knowledge, the work described here is the first quantitative measurement results that have been carried out on a turbulent swirling jet flow using the new dual-plane dye laser PIV technique to analyse large-scale helical structures.

The structure of the present paper is as follows. In the subsequent section, the dynamics and importance of helical structures in fluid flows will be given. The new dual-plane dye laser concept and the experimental setup are then presented. This is followed by the presentation and discussion of experimental results from the dual-plane PIV measurements. This paper then ends with conclusions drawn from this experimental work.

\section{Helical flows}

In industrial flows there is a constant need to improve mixing processes between reactants for an increase in mixing efficiency. For example, in combustion processes, better mixing between fuel and air can reduce the formation of $\mathrm{NO}_{\mathrm{x}}$ levels. Swirling or helical flows can improve mixing and it has been estimated that up to twenty-fold improvement in eddy diffusivity is achievable with helical flows, Zimmerman (1996). The distinction between swirling and helical flow is such that swirling flow is characterised by the rotational motion, whereas helical flow is characterised by the fact that the fluid rotates around an axis that is parallel to the main flow direction.

In turbulent flows, the importance of helicity originates from the facts that it is an inviscid invariant and it is related to the linkage of the vortex lines of the flow, Moffatt and Tsinober (1992). Helicity in fluid flow is defined as the integrated scalar product of the local velocity vector, $\boldsymbol{u}$ and the vorticity vector $\omega$, Eq. 1. Helicity density is defined as the dot product of the local velocity vector and the local vorticity vector, Eq. 2 . Both helicity and helicity density are pseudoscalar quantities, i.e., the quantities will admit different signs, which shows the direction of rotation of the fluid (either clockwise or counter-clockwise). 


$$
\begin{aligned}
\mathscr{H} & =\int h d V \\
h & =\boldsymbol{u} \cdot \boldsymbol{\omega} \\
& =u_{x} \omega_{x}+u_{y} \omega_{y}+u_{z} \omega_{z}
\end{aligned}
$$

It is well known that the vorticity vector is defined as the curl of velocity and can be expressed in the tensor notation in the Cartesian coordinate system as:

$$
\begin{aligned}
\omega_{i} & =\varepsilon_{i j k} \frac{\partial u_{k}}{\partial x_{j}} \\
& =\left[\frac{\partial w}{\partial y}-\frac{\partial v}{\partial z}\right] i+\left[\frac{\partial u}{\partial z}-\frac{\partial w}{\partial x}\right] j+\left[\frac{\partial v}{\partial x}-\frac{\partial u}{\partial y}\right] k
\end{aligned}
$$

where $\varepsilon_{i j k}$ is the alternating tensor and $u_{k}$ is the velocity vector with components of $u, v$ and $w$. The index and component notation that is used to represent vectors will be used in the rest of the paper interchangeably.

Levich and Tsinober (1983a), Levich and Tsinober (1983b) discussed the possibility that helical structures with maximal helicity angle (also termed maximal helicity) should exist in isotropic turbulence at all scales due to a fractal-like creation process. Helical structures with maximal helicity is defined as one where the angle between velocity, $\boldsymbol{u}$ and vorticity, $\omega$ is either $0^{\circ}$ or $180^{\circ}$, (Eq. 4), and is described as being a helical coherent structure (HCS), Zimmerman (1996). In literature, the angle between velocity and vorticity vectors is also referred to as relative helicity or normalised helicity density.

$\cos (\theta)=\frac{\boldsymbol{u} \cdot \boldsymbol{\omega}}{|\boldsymbol{u}||\boldsymbol{\omega}|}$

Moffatt (1985) and Tsinober and Levich (1983) both postulated that flows with regions of relatively high helicity should have low turbulent kinetic energy dissipation rate. The turbulent kinetic energy dissipation rate or, more briefly, energy dissipation, is an important quantity that quantifies the rate at which the energy flux is converted into heat due to the work done by viscous stresses. The energy dissipation is given by:

$$
\begin{aligned}
& \varepsilon=v \overline{\left[\frac{\partial u_{i}}{\partial x_{j}}+\frac{\partial u_{j}}{\partial x_{i}}\right] \frac{\partial u_{j}}{\partial x_{i}}} \\
& =v \overline{\left\{2\left[\left(\frac{\partial u}{\partial x}\right)^{2}+\left(\frac{\partial v}{\partial y}\right)^{2}+\left(\frac{\partial w}{\partial z}\right)^{2}\right]\right.} \\
& +\overline{\left(\frac{\partial v}{\partial x}\right)^{2}+\left(\frac{\partial w}{\partial x}\right)^{2}+\left(\frac{\partial u}{\partial y}\right)^{2}+\left(\frac{\partial w}{\partial y}\right)^{2}+\left(\frac{\partial u}{\partial z}\right)^{2}} \\
& \left.+\overline{\left(\frac{\partial v}{\partial z}\right)^{2}+2\left(\frac{\partial u}{\partial y} \frac{\partial v}{\partial x}+\frac{\partial u}{\partial z} \frac{\partial w}{\partial x}+\frac{\partial w}{\partial y} \frac{\partial v}{\partial z}\right)}\right\}
\end{aligned}
$$

where $v$ is the kinematic viscosity. The overline denotes an ensemble average quantity.
Direct numerical simulations (DNS) of turbulent flow have illustrated interesting and contradictory results. Rogers and Moin (1987) and Kerr (1987) carried out numerical solutions of homogeneous flows and fully developed turbulent channel flows at higher resolutions. The authors concluded that isotropic flows are only slightly helical and found no correlation between structures having maximal helicity and low turbulent kinetic energy dissipation.

Orlandi and Fatica (1997) and Orlandi (1997) carried out direct numerical investigations where the large scale forcing was helical and found the remarkable features that there were both the tendency of $0^{\circ}$ and $180^{\circ}$ helical angle and the correlation between maximal helicity and low energy dissipation. This is an attractive prospect as it implies that effective dispersion of heat or mass using low mechanical energy consumption is possible with the use of helical flows with maximal helicity (helical coherent structures). By contrast, recent 3-D laminar studies of flow through a staggered herringbone microchannel at low Reynolds number conducted by Hassell and Zimmerman (2006) showed the opposite in that an increase in helicity in flows corresponded to an increase in the energy dissipation.

A similar conclusion was found by experimental investigation conducted by Wallace et al. (1992) using a miniature nine-sensor hot-wire probe in a turbulent twostream mixing layer. They found that in turbulent boundary layers, mixing layers, and in grid flow the relative orientation of velocity and vorticity inside the vortex depends on the orientation of the vortex axis relative to the mean flow. They also found little connection between the energy dissipation rate and helicity density. However, they found it difficult to obtain reliable and accurate results as it is well known that hot-wire probes disrupts the fluid flow and can cause anomalous measurement results.

The experimental measurement of helicity density is extremely difficult as the simultaneous measurements of both the velocity and vorticity vectors are required. To measure all three components of vorticity, the dual-plane dye laser PIV is used in this work to generate two light sheets with different wavelengths. The separation of the two different light sheets will allow for the gradients of the velocities to be computed.

In the next few sections, the principle and setup of the dual-plane dye laser system used for the investigation of the turbulent swirling jet will be discussed.

\section{Dual-plane dye laser PIV technique and system setup}

The schematic (perspective view) of the dual-plane dye laser PIV setup and a definition sketch of coordinate system for the measurement area are shown in Figs. 1 and 2. The dual-plane dye laser PIV system consists of a 


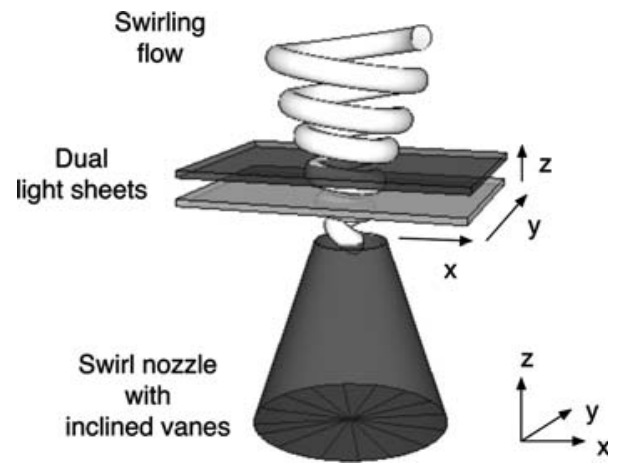

Fig. 1 Definition sketch of coordinate system for the measurement area. The velocity components for $x, y$ and $z$ cartesian coordinates are $u, v$ and $w$ respectively

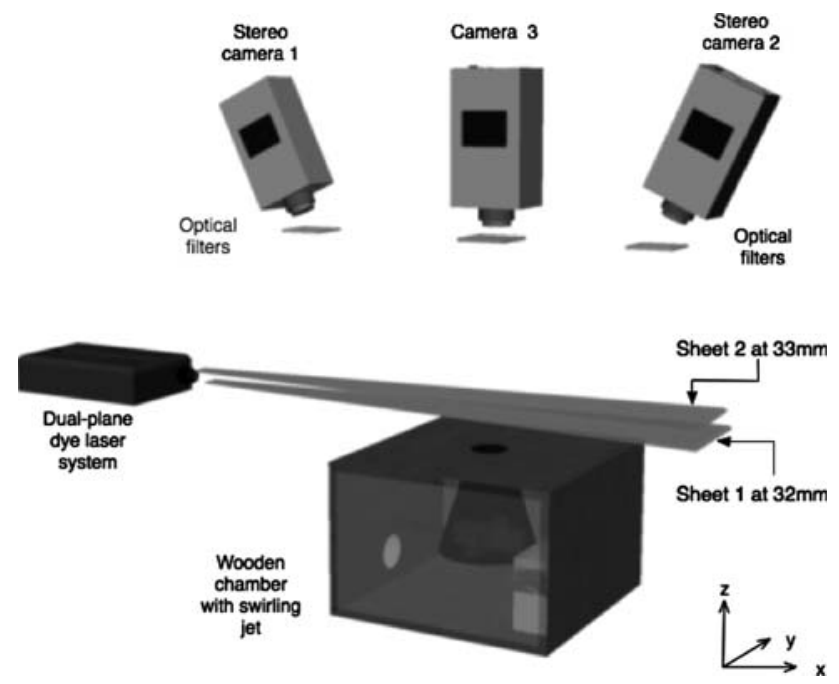

Fig. 2 Perspective view of the dual-plane dye laser PIV system that utilises three cameras, a single Nd:YAG laser and the additional dye laser box to generate two different light sheets

recording system and an illuminating light source. For the recording system, three CCD cameras (PCO Sensicam) are employed to capture the flow field of the measurement area. Unlike other dual-plane PIV systems, only a single $\mathrm{Nd}$ :YAG laser is used in this work to generate two different light sheet wavelengths. However, to generate two different light sheet wavelengths using a single laser source, some optical modifications with the laser have to be made, $\mathrm{Hu}$ et al (2001). The light scattered from particles in the two different light sheets are separated by utilising appropriate optical filters that will only allow a certain wavelength to pass through the CCD camera lens. This technique that has been employed to generate two different light sheets of differing wavelengths using a single laser source is novel and a thorough description of the system will be provided in the following sections.

\subsection{Dye laser system}

The dual-plane dye laser PIV system used in this work uses a single double-pulsed Nd:YAG laser (New Wave, $50 \mathrm{~mJ} /$ pulse) and a dye laser box. The dye laser box is connected to the Nd:YAG laser and comprises of additional optics and a suitable dye to generate the different colour light sheet. This laser system is an experimental prototype, which is now commercialised by Oxford Lasers Ltd, UK.

The laser beam produced by the Nd:YAG laser enters the dye laser box and is separated into two beams by a beam splitter. One of the separated beams is then transmitted through a fluorescent dye, which produces a different beam wavelength to the $\lambda=532 \mathrm{~nm}$ beam generated by the Nd:YAG laser. The wavelength of the beam that is transmitted through the fluorescent dye is $\lambda=572 \mathrm{~nm}$.

One of the major benefits of the dual-plane dye laser system is that it is low in cost and simple in design. It can also be used with most commercial Nd:YAG PIV lasers. The use of one laser source instead of two laser sources contributes to capital equipment savings. In addition, unlike the dual-plane polarisation based technique, the dual-plane dye laser system can be used in reacting and non-reacting flows. This is because with the polarisation based technique the incident polarisation has to be preserved during the scattering process on the tracer particles, Landreth and Adrian (1988). For this, only spherical tracer particles like water and oil droplets can be used. However, this impedes the usage of dual-plane polarisation based technique in exothermic reacting flows where such spherical droplets do not survive. For exothermic reacting flows, solid metal oxide particles like $\mathrm{TiO}_{2}, \mathrm{ZrO}$, $\mathrm{Al}_{2} \mathrm{O}_{3}$ are usually used. These solid metal oxide particles exhibit depolarising effects, which would sharply reduce the signal to noise ratio hence producing noisy PIV images. Therefore the polarisation based PIV technique is not suitable for use in exothermic reacting flows. This problem can be avoided by using two different laser light sheet wavelengths that do not require any polarisations, Mullin and Dahm (2006a).

Having said that however, the advantage with the dualplane polarisation based technique and the two-frequency dual-plane PIV technique developed by Mullin and Dahm (2005) lies in the ability to generate two pairs of light sheets that are spatially (not necessary equally spaced) separated. By spatially separating light sheets, the accuracy of strong out-of-plane flow measurements can be significantly increased, Kähler and Kompenhans (2000). With using a single laser source, it is not possible to independently control the individual spacing of the different coloured light sheets to obtain unequally spaced pair of light sheets. 


\subsection{Recording system and light sheet generation}

The recording system for this work consists of three CCD cameras, PCO Sensicam with a resolution of $1280 \times 1024$ pixels equipped with Nikon $50 \mathrm{~mm}$ lenses that have a minimum $f$-number of 1.8 . Two of the three cameras were setup up using the stereoscopic configuration and the third camera was setup using the standard 2D configuration, refer to Fig. 2. For stereoscopic image recording two basic approaches, which are the lens translation method or the angular displacement method are commonly used. The work described here makes use of the angular displacement configuration with Scheimpflug condition to ensure all particles in the object field will be in good focus in the image field, Prasad and Jensen (1995). Each camera was set at a $45^{\circ}$ angle between the camera axis and the normal to the light sheet, see Fig. 2. For our measurements the $f$-number was set to be 5.6 for all three lenses.

The stereo cameras 1 and 2 are placed on a specially designed one axis tilt-adapter, which is also known as the Scheimpflug adapter. This adapter allows the rotation of the camera, such that the object plane, the camera lens plane and the image plane all intersect at a common point. These two cameras (cameras 1 and 2) were fitted with $540 \mathrm{~nm}$ shortpass optical filters that only allowed the stereoscopic measurements on the green light sheet, $\lambda=532 \mathrm{~nm}$ ( $2 \mathrm{~mm}$ in thickness) to be captured and blocked out the $\lambda=572 \mathrm{~nm}$ wavelength. Camera 3 was fitted with a $550 \mathrm{~nm}$ longpass optical filter that only permitted 2D measurements on the yellow light sheet, $\lambda$ $=572 \mathrm{~nm}$ ( $2 \mathrm{~mm}$ in thickness $)$ to be captured and blocked the $\lambda=532 \mathrm{~nm}$ wavelength. These filters isolated the scattered light from the two different laser light sheets wavelengths onto appropriate cameras. The three CCD cameras and the double-pulsed Nd:YAG laser were connected to a synchroniser, which controlled the timing of the laser light sheets illumination and image acquisition.

The two light sheets generated by the dual-plane dye laser system consist of a green light sheet, $\lambda=532 \mathrm{~nm}$ and a yellow light sheet, $\lambda=572 \mathrm{~nm}$. The adjustment of the displacement between the two light sheets can be easily done by the rotating mirror in the dye laser box. By adjusting the mirror appropriately, the two light sheets can coincide with each other or be separated by a particular distance.

In the present study, each laser light sheet pair is aligned independently to illuminate a specific $z$ location. The thickness and separation of the two lights sheets were determined by using a camera that was placed in parallel with the Nd:YAG laser. A piece of dark sheet was placed in the measurement area and the lasers were triggered. The image of the two laser light sheet planes that was seen on the paper were captured and analysed. The thickness of each light sheet was found to be approximately $2 \mathrm{~mm}$ and the centres of the two light sheets were located at $32 \mathrm{~mm}$ and $33 \mathrm{~mm}$. Therefore, the distance between the centres of the two overlapping light sheets is $1 \mathrm{~mm}$. Although these two light sheets overlapped, the optical filters that were placed in front of the cameras only permitted one of the two wavelengths to be captured. To generate the plane of light sheet, suitable cylindrical and spherical optical lenses were used.

At each centre positions of the two light sheets, $z=32 \mathrm{~mm}$ and $z=33 \mathrm{~mm}$, the image calibration procedure has to be carried out accordingly. For the calibration procedure, which will be discussed in the next section, a two-axis translation scheme of $1 \mathrm{~mm}$ accuracy was used. Due to the resolution of the two-axis translation system, the distance between the centres of the two light sheets was therefore chosen to be $1 \mathrm{~mm}$ apart to permit the accurate calibration process.

\subsection{Image calibration and processing}

The reconstruction procedure is the process of mapping the displacements from each image plane of the respective CCD stereo camera to the object plane and combining them to obtain the three-dimensional data. The reconstruction procedure is crucial as it allows the determination of positions (in real dimensions) in the object plane to be mapped to the positions (in pixels) in the image plane.

The calibration procedure for the stereo cameras 1 and 2 was based on the 3-D calibration-based reconstruction method following the work of Soloff et al (1997) for the stereoscopic plane. This particular method takes into account the distortions in images that are caused by nonuniform magnification. Moreover, with this particular calibration technique, a priori knowledge of the recording geometry is not required.

The calibration procedure was carried out by taking images of a calibrated target where the positions of the target are known. In this work, a calibration target with $1 \mathrm{~mm}$ diameter dots spaced at intervals of $2 \mathrm{~mm}$ was placed in the object plane, which coincided accurately with the laser light sheet. Once the calibration target was accurately placed in the object plane, the two stereo cameras were used to capture the calibration images at three different locations across the depth of the laser light sheet. For accurate and precise calibration of the images at three different locations, a two-axis translation scheme of $1 \mathrm{~mm}$ accuracy was utilised. Images of the calibration target were acquired by each of the stereo camera to estimate the mapping functions using least squares polynomial approach. These polynomial functions are cubic in the $x$ and $y$-directions and quadratic in the $z$-direction.

For camera 3 which would capture two-dimensional data, the calibration procedure was based on the conventional 
calibration procedure of using a calibration grid of known dimensions. The calibration grid used was the same as the calibration grid used for the 3-D calibration procedure. However, instead of capturing calibration images at three different locations, only a single calibration image was captured. By identifying the positions of points on the image and combining these with their known real positions, a linear mapping is generated using a least squares fit. This mapping actually scales between pixels and real dimensions.

The PIV images were divided into $32 \times 32$ pixel window and were interrogated with $50 \%$ overlap to allow cross-correlation processing using the fast Fourier transform (FFT) algorithm. This corresponded to a spatial resolution of $2 \mathrm{~mm}$ in each direction of physical space. The PIV software provided by Oxford Lasers was used for both the reconstruction procedure and image analysis. For this study, the time interval between the two illuminations of the pulsed laser was set to be $45 \mu$ s.

The resultant vector fields were filtered using both the global and local filters that identifies the global inconsistent values and local variations in vector magnitude and direction respectively. Missing vectors were then interpolated using a $3 \times 3$ local mean technique. The number of spurious vectors was less than $5 \%$ in the stereo plane and less than $3 \%$ in the single plane. Once the cross-correlation procedure was performed, the data was then exported to MATLAB software created by The MathWorks for further data processing and analysis. In MATLAB, the vector fields from the single camera is resampled and mapped to the grid of the stereo measurements using the two-dimensional linear interpolation (also known as bilinear interpolation) method.

\subsection{Tracer particles}

The flow was seeded upstream in the swirling jet chamber with water droplets generated using an ultrasonic humidifier. The ultrasonic humidifier uses a transducer, which oscillates at high frequency (ultrasonic frequency). This high frequency creates the fine water droplets that are released in a form of a cool water mist which are nonirritant, non-flammable and non-hazardous. The generator of water droplets was placed inside the wooden chamber along with the swirl nozzle to generate uniform seeding in the chamber.

From measuring the water particle diameter, a distribution with a mean of approximately $10 \mu \mathrm{m}$ was obtained. The particle settling velocity and Reynolds number was calculated and it was found that the particle settling velocity was approximately $3.04 \mathrm{~mm} \mathrm{~s}^{-1}$. According to Prasad (2000), as long as the particle settling velocity is negligible when compared to the actual flow velocities, the particles are suitable.

\section{Swirling jet}

To investigate and generate fluid flow of a helical nature, a swirling nozzle imposing both a tangential and axial momentum within the fluid flow was designed and used. The swirl nozzle used in this study uses the principle of fixed vanes set at an inclination to impart the tangential motion in the flow. The swirl nozzle is located in the wooden chamber and has an exit diameter of, $D=32 \mathrm{~mm}$ which is open to the surroundings. The direction of the airflow is in the vertical direction (streamwise direction). Pressurised air was supplied by a centrifugal compressor through flow meters and valves. The measurement area was at the top of the nozzle exit, one diameter away from the nozzle exit at the $32 \mathrm{~mm}$ plane.

The Swirl number, $S$, as defined by Gupta et al. (1984), is a non-dimensional number which is used to characterise the degree of swirl in the flow. It represents the axial flux of tangential momentum divided by the axial flux of axial momentum times the equivalent nozzle radius.

$S=\frac{\int_{0}^{R} u w r^{2} \mathrm{~d} r}{R \int_{0}^{R} u^{2} r \mathrm{~d} r}$

where $R$ represents the radius of the swirl nozzle, $u$ is the axial velocity, $w$ is the tangential velocity and $r$ is the radial coordinate.

In the present study, the jet velocity was measured to be $5,130 \mathrm{mms}^{-1}$. The corresponding Swirl number and Reynolds number based on the swirl nozzle exit diameter are $S=0.44$ and $R e=11,890$, respectively. The Swirl number was computed by numerically integrating the axial and tangential velocity profiles.

Using the dual-plane dye laser PIV system, the largescale helical structures in the swirling jet can be investigated and analysed from the velocity measurements. The analysis of these large-scale helical structures will be described and presented in Sect. 6.

\section{Measurement uncertainties}

An uncertainty analysis was carried out to quantify the accuracy of the velocity gradients. It is well known that the uncertainty of the computed velocity gradient can be divided into two components, Lourenco and Krothapalli (1995). One is the truncation error associated with the finite difference scheme used and the other is due to the uncertainty in the velocity measurements. 
By applying the error propagation analysis described by Kline and McClintock (1953), the relative errors in the velocity gradients were computed. Table 1, shows the computed relative uncertainties in all nine gradients. The relative uncertainty (column 3) was computed by normalising the absolute uncertainty of each gradient with the r.m.s (root mean square) values of the particular gradients, Ganapathisubramani et al. (2005).

The uncertainties in both the first order difference scheme and in the sheet separation along the $z$-axis causes a significant increase in the uncertainties of $\partial u / \partial z$ and $\partial v / \partial z$. Therefore, the absolute uncertainty in these two particular gradients are the largest relative to the in-plane gradients $\partial u / \partial x, \partial v / \partial x, \partial u / \partial y$ and $\partial v / \partial y$. The largest relative uncertainty however is observed for the $\partial w / \partial z$ gradient. This high relative uncertainty may initially be surprising since $\partial w / \partial z$ is computed from $\partial u / \partial x$ and $\partial v / \partial y$, which themselves are determined more accurately, however, since the r.m.s value of $\partial w / \partial z$ is small, the relative uncertainty is amplified. The relative uncertainties in the vorticity components $\omega_{x}, \omega_{y}$ and $\omega_{z}$ were 19,16 and $6 \%$ respectively. For helicity, the relative uncertainty was found to be approximately $13 \%$.

\section{Experimental results and discussion}

The dual-plane dye laser PIV technique was used to illuminate the $32 \mathrm{~mm}$ and $33 \mathrm{~mm}$ spanwise planes to measure the fluid velocities. The single camera vector field from the upper plane, $33 \mathrm{~mm}$, in liaison with the stereoscopic vector field from the lower plane, $32 \mathrm{~mm}$, are then used to compute all velocity gradients in the lower plane. The velocity gradient fields were obtained by second-order central differences of the measured velocity component values within each plane and first-order forward difference between the two planes separated in the $z$ direction.

Table 1 Uncertainty analysis of velocity gradients

\begin{tabular}{lcc}
\hline$G=\partial u_{k} / \partial x_{j}\left(\mathrm{~s}^{-1}\right)$ & $\sigma_{G}\left(\mathrm{~s}^{-1}\right)$ & $\left(\sigma_{G} / G_{\mathrm{rms}}\right) \%$ \\
\hline$\partial u / \partial x$ & 5.24 & 16.9 \\
$\partial v / \partial x$ & 4.86 & 10.3 \\
$\partial w / \partial x$ & 10.92 & 19.0 \\
$\partial u / \partial y$ & 5.24 & 8.3 \\
$\partial v / \partial y$ & 4.86 & 15.4 \\
$\partial w / \partial y$ & 10.92 & 14.7 \\
$\partial u / \partial z$ & 21.34 & 16.7 \\
$\partial v / \partial z$ & 19.84 & 19.1 \\
$\partial w / \partial z$ & 7.14 & 27.4
\end{tabular}

Column 1 reflects the velocity gradient components, column 2 shows the absolute uncertainty in the gradient and column 3 shows the relative uncertainty in the gradients
The measurement results of the dual-plane dye laser PIV system was based on the ensemble time-average of 500 image pairs. The instantaneous velocity vectors were averaged to produce mean velocity vectors at each position. The velocity measurements results of the two planes $(z=33 \mathrm{~mm}$ and $z=32 \mathrm{~mm}$ ) away from the nozzle exit are shown in Figs. 3 and 4, respectively. These figures show the average velocity vectors obtained using the three camera dual-plane PIV setup. Figure 3 shows the average two-dimensional velocity vectors $\bar{u}$ and $\bar{v}$ captured at $z=33 \mathrm{~mm}$ using a single CCD camera. At $z=32 \mathrm{~mm}$, the stereoscopic CCD camera arrangement was used to capture the three-dimensional velocity field and thus the averaged velocity vectors shown in Fig. 4 are the $\bar{u}, \bar{v}$ and $\bar{w}$ velocities.

Figure 5 shows the contours of the average velocity magnitude in $\mathrm{mms}^{-1}$ and the superimposed velocity vector plot at the $32 \mathrm{~mm}$ plane. All contour plots have 10 equally spaced contour levels between the maximum and minimum values. By observing the direction of the velocity vectors in the figure, it can be clearly seen that the flow is rotating in the counter clockwise direction. The contours of the velocity magnitude suggests that the velocity increases radially until it reaches the maximum and then decays further away from the nozzle. This can be observed clearly in Figs. 6 and 7, where the plot of the mean axial velocity and mean tangential velocity normalised by the centreline velocity, $U_{C}$ across the horizontal dotted centreline (shown

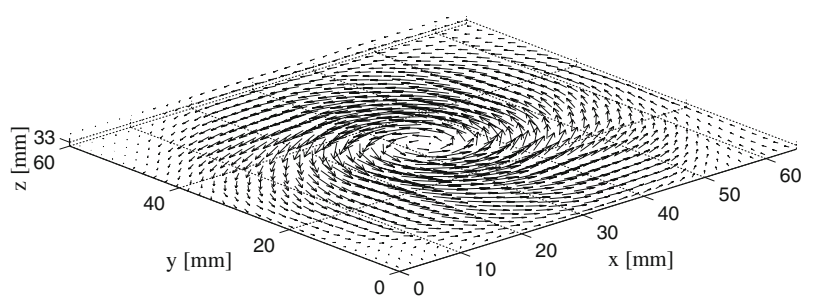

Fig. 3 The $z=33 \mathrm{~mm}$ plane was illuminated with a light sheet of wavelength, $\lambda=572 \mathrm{~nm}$ and using a single CCD camera the average $2 \mathrm{D}$ velocity vectors, $\bar{u}$ and $\bar{v}$ for the $z=33 \mathrm{~mm}$ plane were captured. The circulation can be clearly observed in the $2 \mathrm{D}$ plane

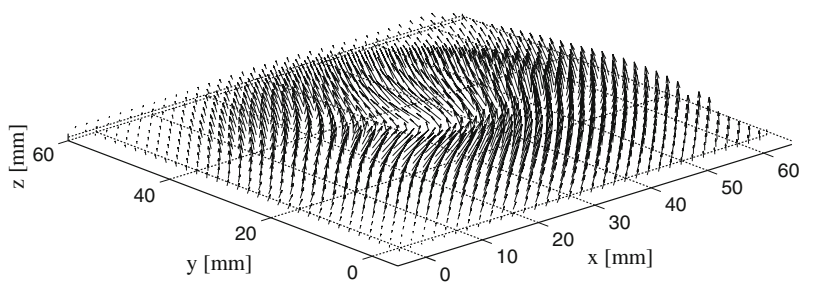

Fig. 4 The $z=32 \mathrm{~mm}$ plane was illuminated with a light sheet of wavelength, $\lambda=532 \mathrm{~nm}$ and using the stereoscopic CCD camera arrangement the average $3 \mathrm{D}$ velocity vectors, $\bar{u}, \bar{v}$ and $\bar{w}$ for $z=32 \mathrm{~mm}$ plane were captured. In contrast to Fig. 3 , the vortex has a strong localised $3 \mathrm{D}$ character 


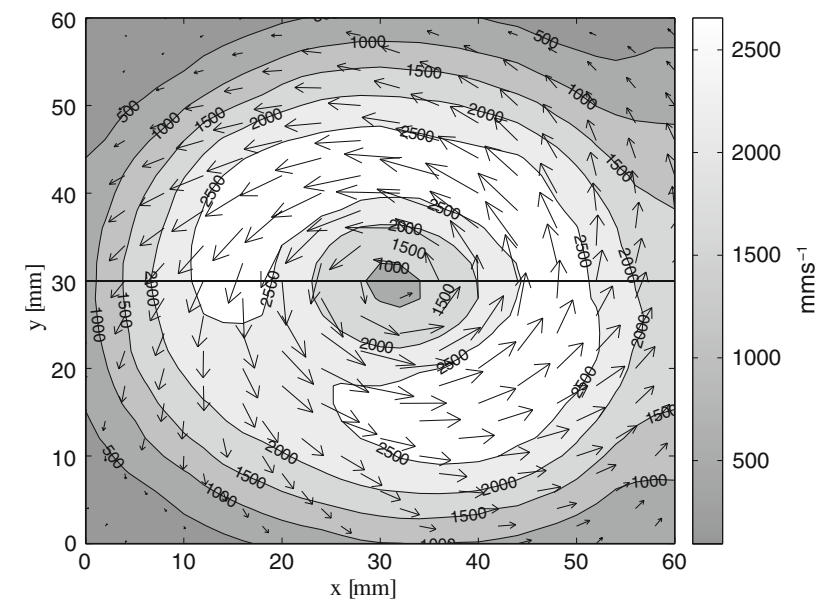

Fig. 5 Contours of average velocity magnitude and the superimposed average velocity vector plot at the $z=32 \mathrm{~mm}$ plane. The velocity vectors show that the fluid is rotating in the counter clockwise direction

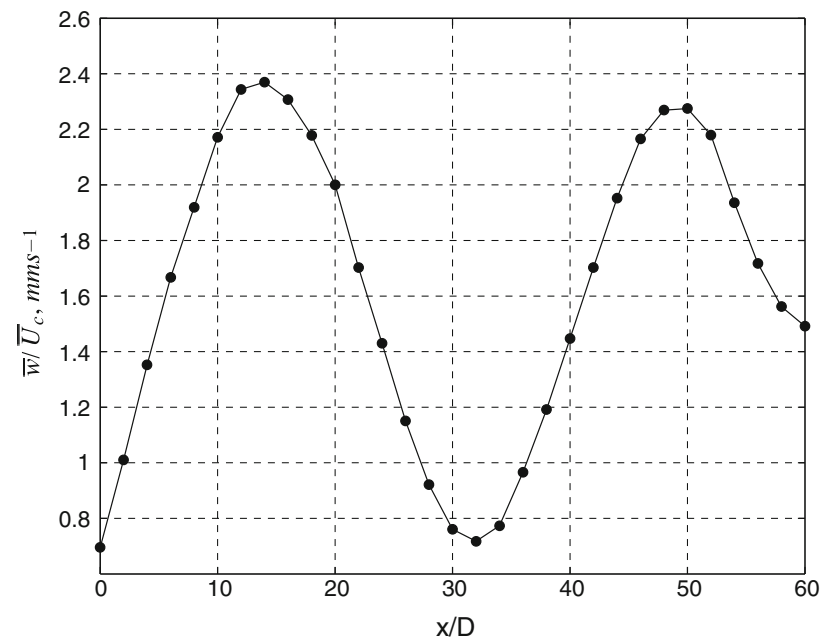

Fig. 6 Plot of normalised mean axial velocity across the centreline of the nozzle. The velocity profile has the shape of double crests separated by a central trough

in Fig. 5) are shown. The mean axial velocity profile shown has the shape of double crests separated by a central trough while the mean tangential velocity profile shows the solid body rotation flow where the velocity decreases as the distance from the centre increases. These velocity profiles are in good agreement with previously reported results for swirling air jet from vane swirlers, (Mathur and Maccallum (1967); Panda and McLaughlin (1994)).

To compute the vorticity vector, $\omega$, all the vorticity components $\omega_{x}, \omega_{y}$ and $\omega_{z}$ are required, see Eq. 3. Based on the present system, the present dual-plane dye laser PIV system can provide all three components of the velocity fields at the $z=32 \mathrm{~mm}$ plane and two components of the velocity field at the $z=33 \mathrm{~mm}$ plane simultaneously, see

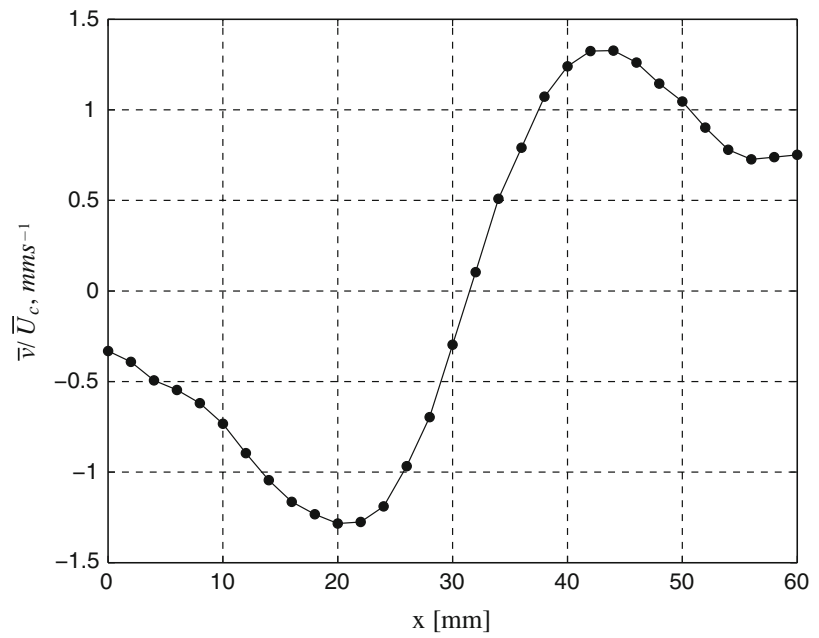

Fig. 7 Plot of normalised mean tangential velocity across the centreline of the nozzle. The tangential velocity profile indicates the solid-body rotation where the velocity drops off with distance from the centre

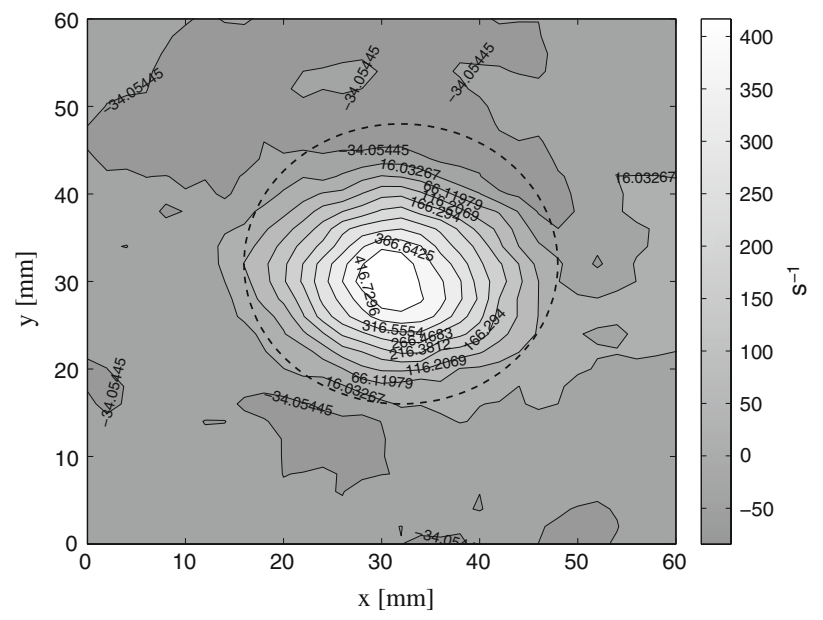

Fig. $8 \bar{\omega}_{z}$ vorticity component for $z=32 \mathrm{~mm}$ plane

Figs. 3 and 4. Since all the three components of the velocity fields at $z=32 \mathrm{~mm}$ can be measured, all the gradients $\partial u_{k} / \partial x_{j}$ can be computed readily and therefore all the vorticity components can be determined for the $z=32 \mathrm{~mm}$ plane. This is not the case for the $z=33 \mathrm{~mm}$ plane, where only the $\omega_{z}$ component of vorticity (out-ofplane) can be computed.

The contour plot of the $\bar{\omega}_{z}$ component of vorticity for the $z=32 \mathrm{~mm}$ is shown in Fig. 8. From this figure it can be seen that the maximum out-of-plane rotation occurs in the centre of the nozzle, which then reduces radially away from the centre of the nozzle. The dashed circle superimposed in the figure represents the swirl nozzle diameter.

The $\omega_{x}$ and $\omega_{y}$ vorticity components involves the determination of the $\partial w / \partial y$ and the $\partial w / \partial x$ gradient terms. These terms can be determined at the plane where the $w$ 
velocity components can be measured, which is the $z=32 \mathrm{~mm}$ plane. Figures 9 and 10 shows the contours of both the $\bar{\omega}_{x}$ and $\bar{\omega}_{y}$ vorticity components for the $z=32 \mathrm{~mm}$ plane.

The contour plot of the average vorticity magnitude for the $32 \mathrm{~mm}$ plane is shown in Fig. 11. From this figure it can be seen that the vorticity magnitude is concentrated in large regions of positive vorticity, where the maximum occurs at the centre of the swirl nozzle.

Since the velocity and vorticity vectors for the $z=32 \mathrm{~mm}$ plane can be computed, the average helicity density, $\bar{h}=\overline{\boldsymbol{u} \cdot \boldsymbol{\omega}}$ that involves these terms can therefore be determined. The plot of average helicity density for the $z=32 \mathrm{~mm}$ plane is shown in Fig. 12. As mentioned in Sect. 2, the helicity density term is a pseudo-scalar term whereby, the sign changes according to the right-handed (positive) or left-handed (negative) chirality. From this figure it can be seen that the helicity density is largely

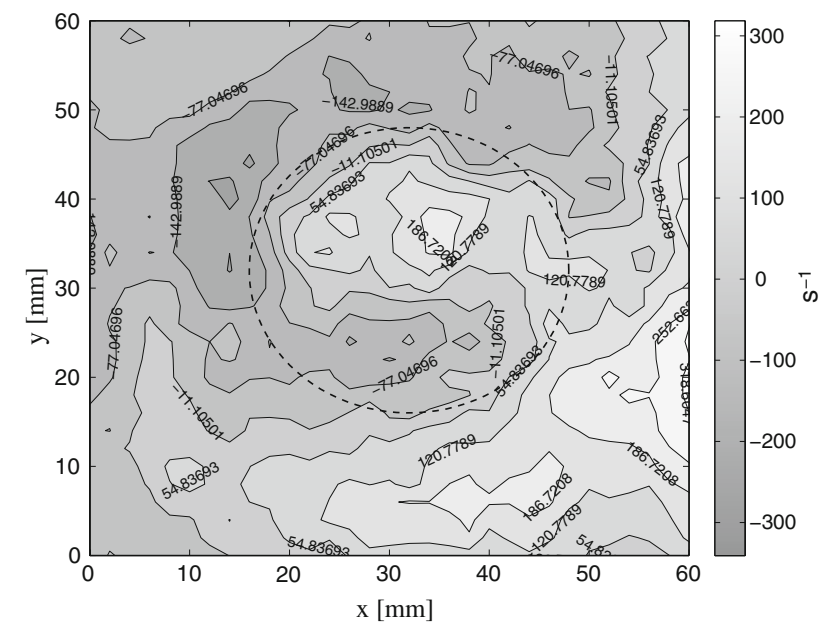

Fig. $9 \bar{\omega}_{x}$ vorticity component for $z=32 \mathrm{~mm}$ plane

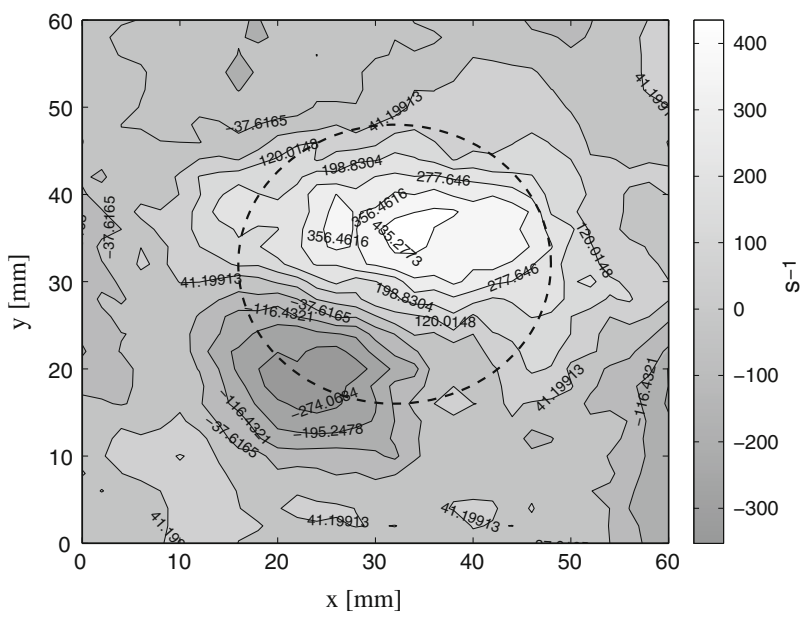

Fig. $10 \bar{\omega}_{y}$ vorticity component for $z=32 \mathrm{~mm}$ plane positive in the $z=32 \mathrm{~mm}$ plane which implies that the chirality of the helical structure is right-handed.

Computing the angle between the vorticity and velocity vector would reflect the regions of maximal helicity, Eq. 4. Figure 13 shows the average angle in degrees, $\bar{\theta}$, between the velocity and vorticity component in the $z=32 \mathrm{~mm}$ plane. For maximal helicity, the angle between vorticity and velocity should be either $0^{\circ}$ (parallel) or $180^{\circ}$ (antiparallel). It is evident from Fig. 13, that hardly any angles of $0^{\circ}$ and $180^{\circ}$ occur in the $z=32 \mathrm{~mm}$ plane. To elucidate the occurrence of maximal helicity, a histogram of the frequency of average helicity angle is shown in Fig. 14. In addition to the histogram of the frequency of average helicity angle, a histogram illustrating the occurrences of helicity angle from all 500 images is also shown in Fig. 15.

Both these histograms further elucidates the observation that there are hardly any evidences of the $0^{\circ}$ and $180^{\circ}$

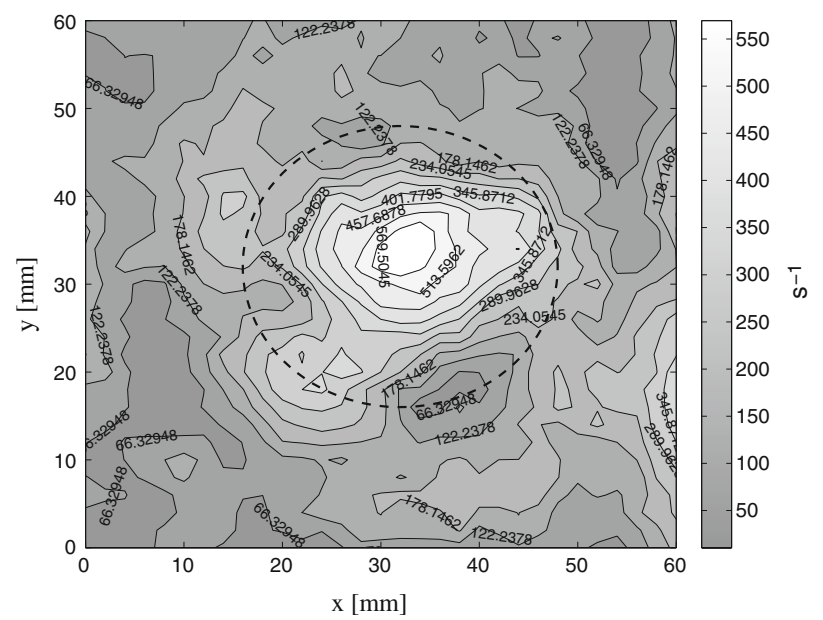

Fig. 11 Average vorticity magnitude for $z=32 \mathrm{~mm}$ plane

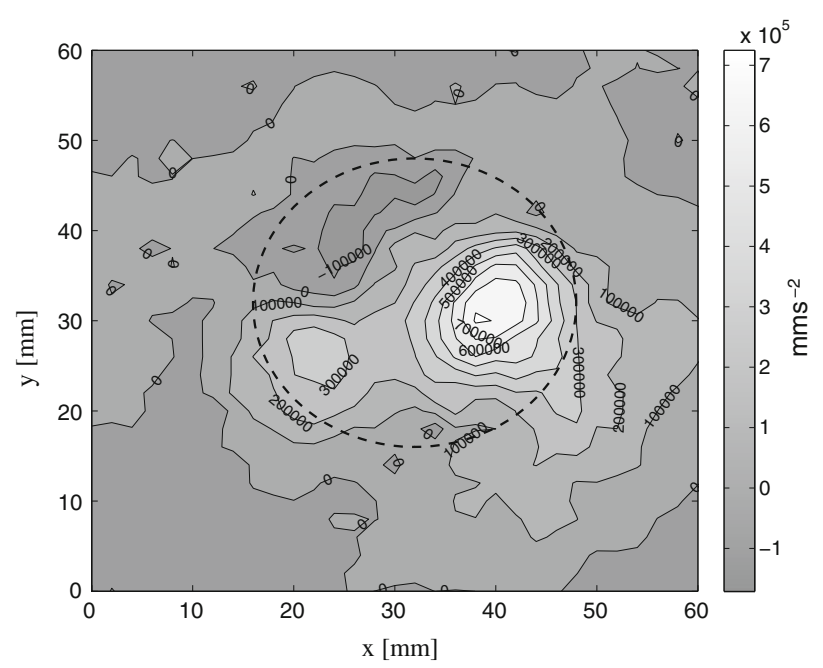

Fig. 12 Average helicity density, $\bar{h}$ plot for $z=32 \mathrm{~mm}$ plane showing concentrated regions of high helicity density 


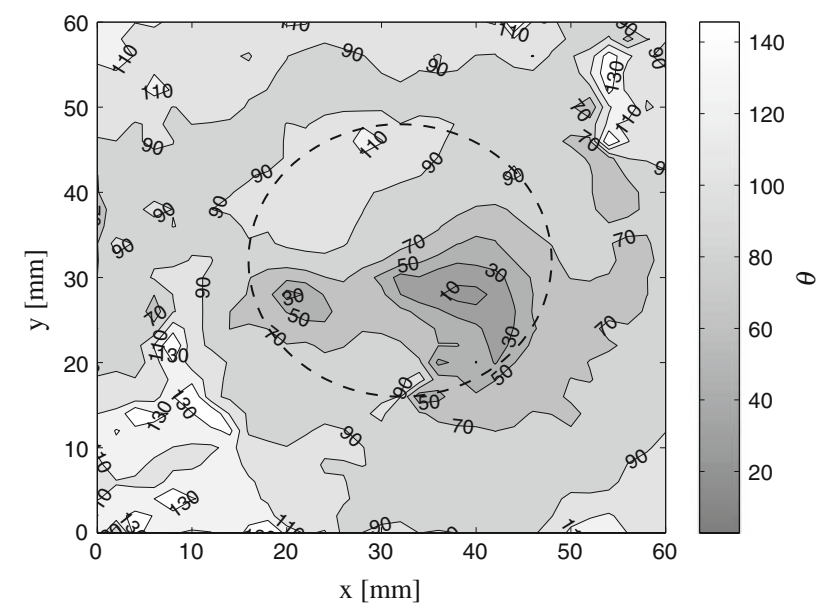

Fig. 13 This figure illustrates the plot of average helicity angle, $\bar{\theta}$ in degrees for $z=32 \mathrm{~mm}$ plane. This plot shows areas with close to $0^{\circ}$ within the diameter of the swirl nozzle indicating the possibility of the vorticity and velocity vectors being parallel

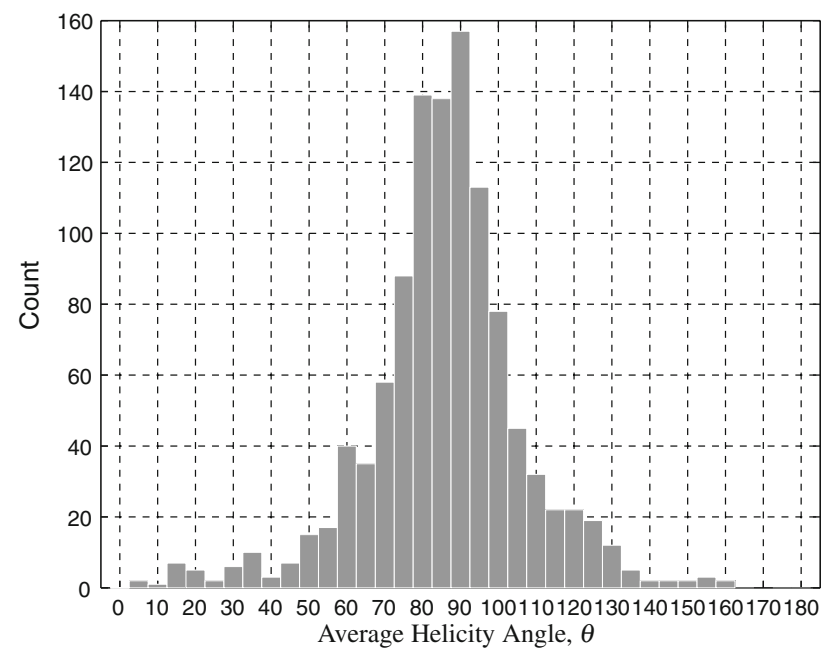

Fig. 14 This figure shows the frequency of average helicity angle for $z=32 \mathrm{~mm}$ plane. From this figure it can be seen the preferred alignment for the vorticity and velocity vectors is to be orthogonal $90^{\circ}$

angles, which implies that there are no occurrences of maximal helicity in the $32 \mathrm{~mm}$ plane. In fact, the highest occurring angle is the $90^{\circ}$ angle, which implies that the vorticity and velocity vectors are orthogonal and not parallel.

To analyse the turbulent kinetic energy dissipation rate all the components of the velocity gradients have to be computed, Eq. 5. However with the current dual-plane dye laser PIV setup, only eight out of the nine velocity gradient components can be computed. This is because the velocity component in the $z$-direction, is only being measured on the $z=32 \mathrm{~mm}$ plane, and therefore the gradient $\partial w / \partial z$ cannot be computed. Nevertheless, by using the continuity equation, the $\partial w / \partial z$ can be inferred, Eq. 7.

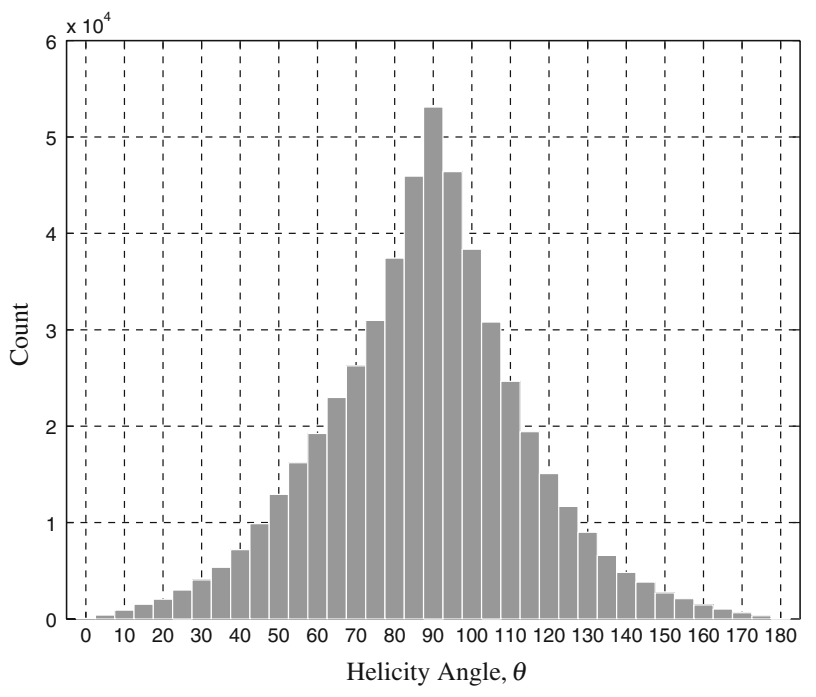

Fig. 15 This figure shows the frequency of helicity angle for $z=32 \mathrm{~mm}$ plane that was computed from all 500 images. From this figure it can be seen the preferred alignment for the vorticity and velocity vectors is also $90^{\circ}$

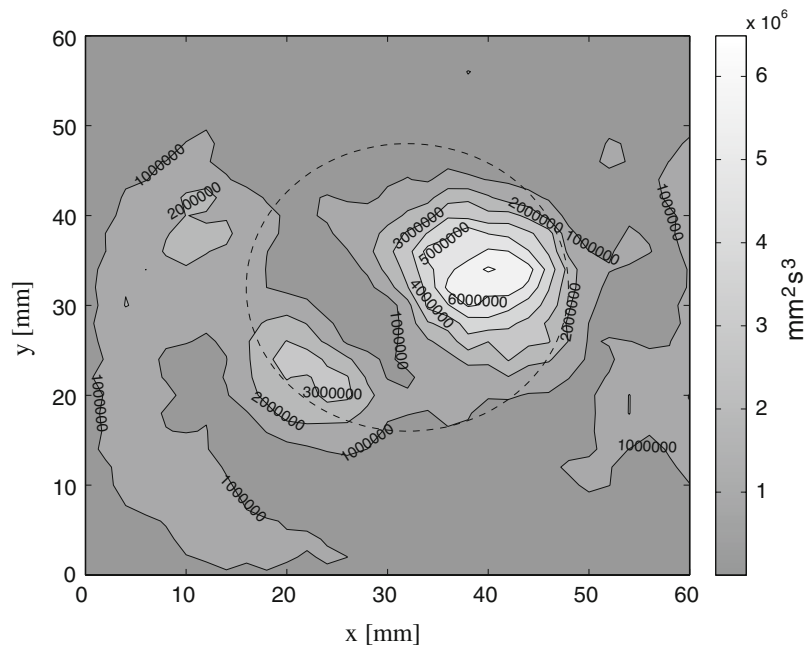

Fig. 16 Plot of average energy dissipation for $z=32 \mathrm{~mm}$ plane showing circular regions of high and low energy dissipation rates

$$
\begin{aligned}
& \nabla \cdot \boldsymbol{u}=0 \\
& {\left[\frac{\partial u}{\partial x}+\frac{\partial v}{\partial y}+\frac{\partial w}{\partial z}\right]=0}
\end{aligned}
$$

Figure 16, shows the plot of the average energy dissipation, $\bar{\varepsilon}$, for $z=32 \mathrm{~mm}$ plane. From this plot, circular regions of relatively high and low energy dissipation were observed and it was in the interest of the authors to analyse the relation between energy dissipation and helicity properties. The analysis of how the average energy dissipation, $\bar{\varepsilon}$ varies with average helicity density, $\bar{h}$, and average helicity angle, $\bar{\theta}$, are depicted in Figs. 17, 18 and 19. Both the 


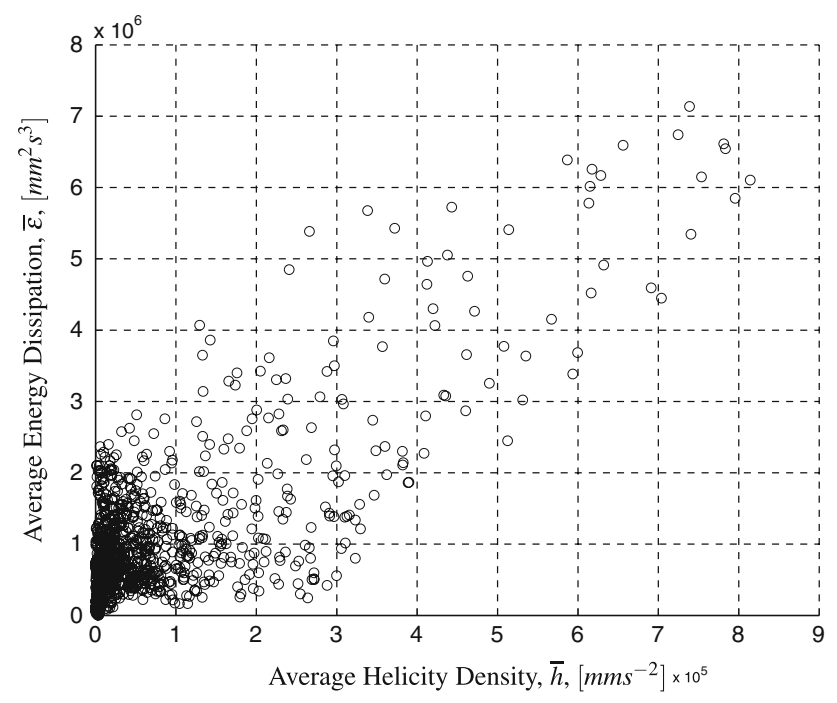

Fig. 17 Scatter plot of average energy dissipation and average helicity density for $z=32 \mathrm{~mm}$ plane showing a positive trend where the kinetic energy dissipation increases with increasing helicity density

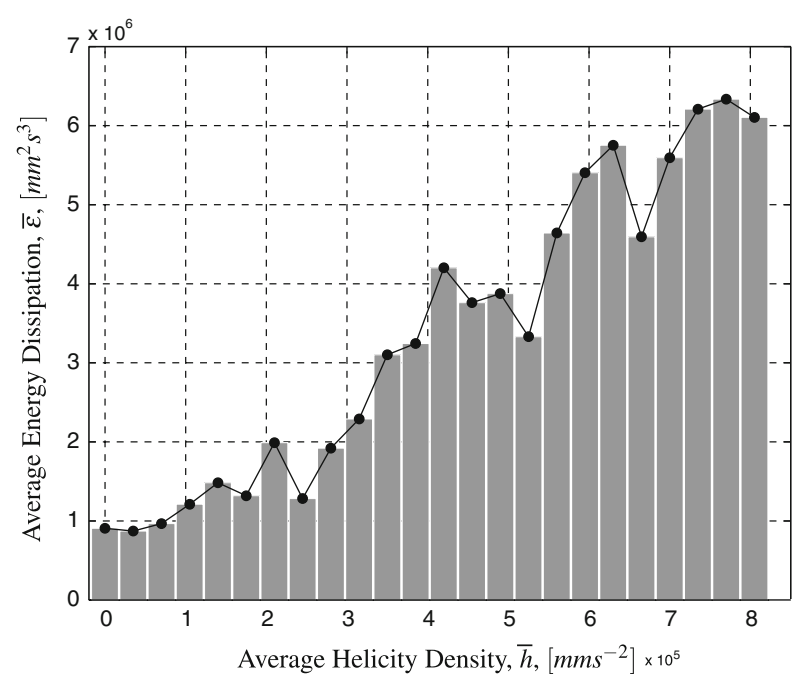

Fig. 18 This figure shows the distribution of average of energy dissipation for the average helicity density at the $z=32 \mathrm{~mm}$ plane. This figure shows that where the average energy dissipation is high, the average helicity density is high

Figs. 17 and 18 show that the average energy dissipation, $\bar{\varepsilon}$ increases with the average helicity density, $\bar{h}$.

The variation of average energy dissipation with average helicity angle in Fig. 19 shows that the average energy dissipation, $\bar{\varepsilon}$ is maximum for angles where the vorticity and velocity vectors are small and close to $0^{\circ}$. For angles where the vorticity and velocity vectors are orthogonal, $90^{\circ}$, the energy dissipation is minimum. From these plots, it is clearly evident that the energy dissipation increases with increasingly pure helicity (defined as helical angle).

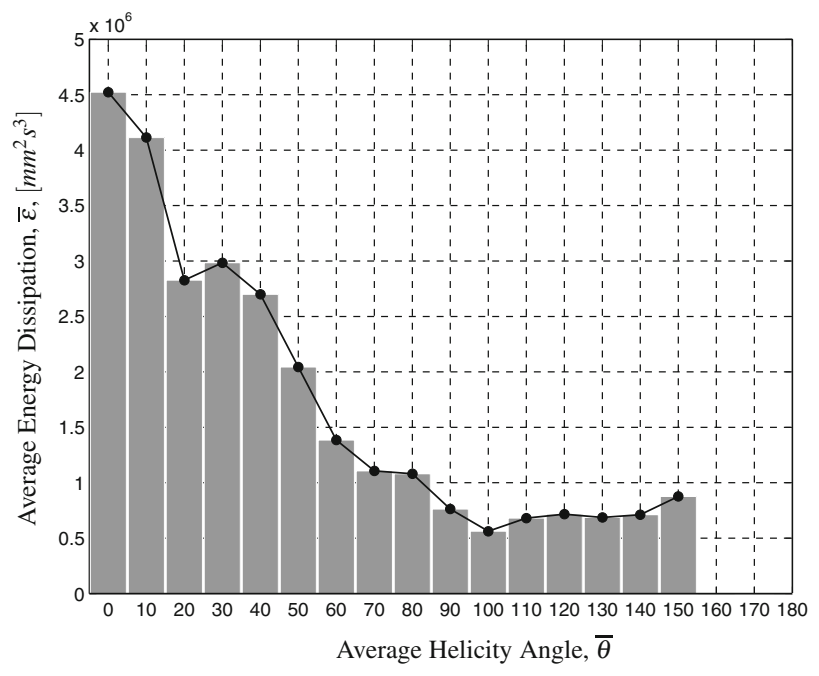

Fig. 19 This figure shows the distribution of average of energy dissipation for the average helicity angle at the $z=32 \mathrm{~mm}$ plane. This figure shows that where the average energy dissipation is high, the vorticity and velocity vectors are parallel and where the average energy dissipation is low, the vorticity and velocity vectors are orthogonal

Since Fig. 14 shows no population of the $0^{\circ}$ or $180^{\circ}$ helical angles, there is no evidence for the existence of large scale helical structures with maximal helicity (vorticity is parallel to velocity). Therefore the conjecture that these structures coincide with overall lower energy dissipation could not be tested.

In our PIV experimental results, the resolution simply does not exist to consider the scales of turbulence just above the Kolmogorov scale. We speculate that it is the limitation to large scale resolution that makes finding these structures so elusive. It is commonly understood in atmospheric dynamics that the large eddy motions are practically inviscid; viscous dissipation is prevalent in much smaller scales. Thus the argument of Levich and Tsinober (1983a) that such long lived helical structures are prevalent is stronger at smaller scales where viscous dissipation is more prominent.

To illustrate the effect of this resolution issue on the estimates of helical coherent structures, a simple calculation was carried out. The ABC (Arnold-BeltramiChildress) flow which satisfies the Beltrami property (that its vorticity is parallel to its velocity field) was setup and analysed. The vorticity fields were estimated using four different resolutions of $d x_{j}=0.2,0.1,0.05$ and 0.025 . For each resolution, the helical angles were computed. The four histograms shown in Figs. 20, 21, 22 and 23 clearly demonstrate the effect of resolution on the occurrences of helical coherent structures. At low resolution, the distribution of helical angles spreads away from the $0^{\circ}$ angle, whilst at higher resolutions the the distribution of helical angles approaches a spike at $0^{\circ}$. This implies that grid 


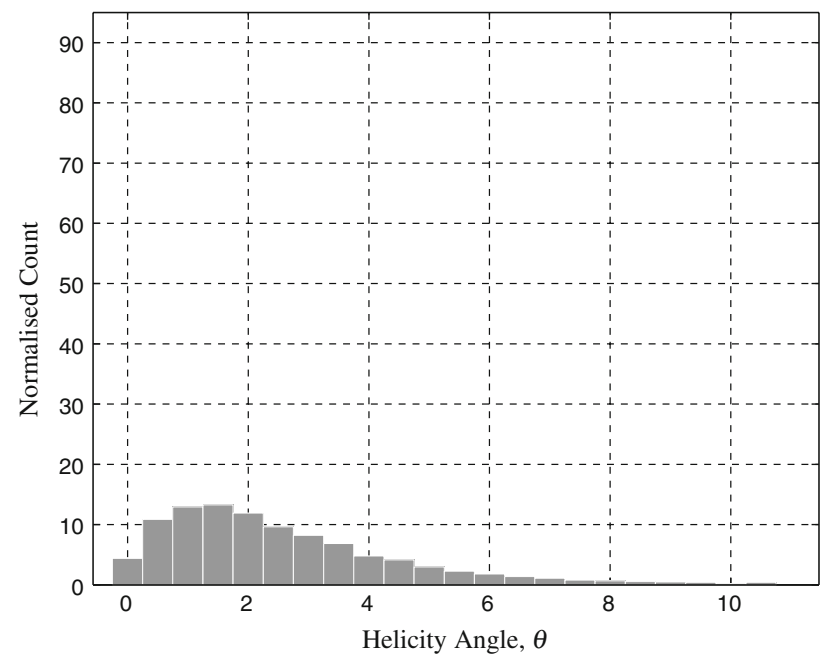

Fig. 20 Histogram showing the frequency of the helical angle computed using a resolution, $d x_{j}=0.2$ for the $\mathrm{ABC}$ flow field that was set up to analyse the effect of resolution on helical coherent structures. At lower resolutions, the distribution of helical angles spreads away from the $0^{\circ}$

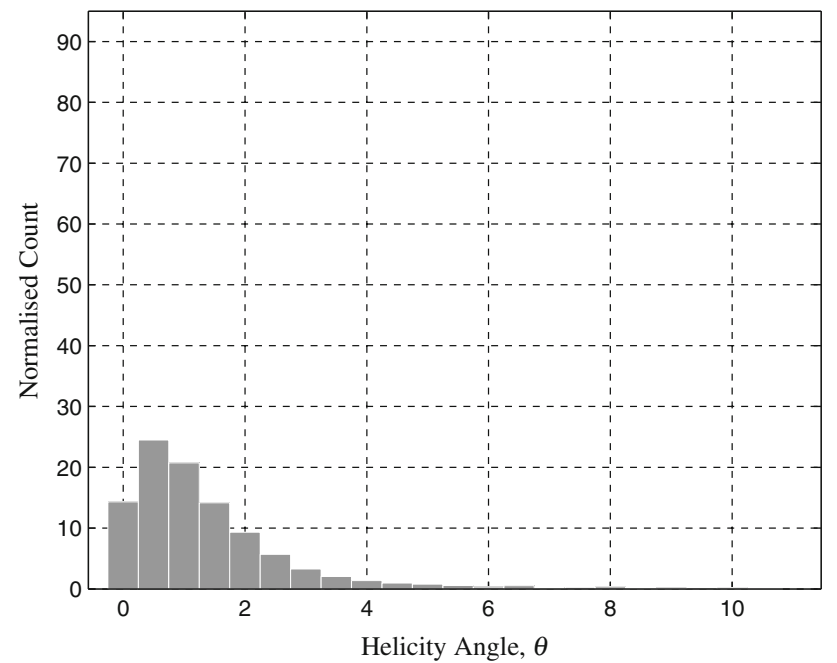

Fig. 21 Histogram showing the frequency of the helical angle computed using a resolution, $d x_{j}=0.1$ for the $\mathrm{ABC}$ flow field that was set up to analyse the effect of resolution on helical coherent structures

resolution used to compute vorticity has a significant affect on the estimation of helical coherent structures reaffirming our speculation.

The theories of Moffatt (1985) and Levich and Tsinober (1983a), Levich and Tsinober (1983b) which predict that regions of high helicity density should have low energy dissipation rate are based on the assumption that helical flows will reorganise themselves into a state of maximal helicity (parallel or antiparallel vorticity and velocity as in Eq. 4). The argument these authors give as to why this should occur is that flows that are maximally helical have

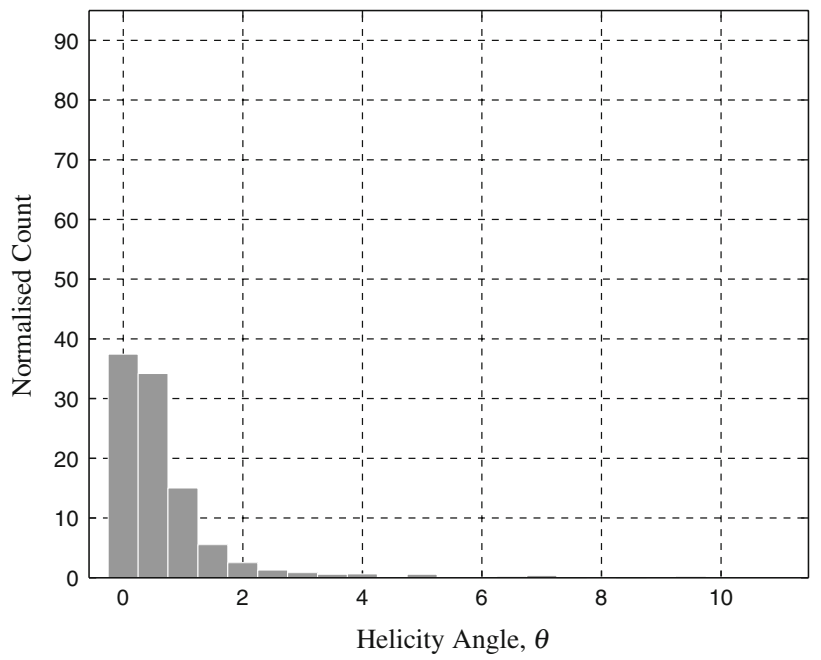

Fig. 22 Histogram showing the frequency of the helical angle computed using a resolution, $d x_{j}=0.05$ for the $\mathrm{ABC}$ flow field that was set up to analyse the effect of resolution on helical coherent structures

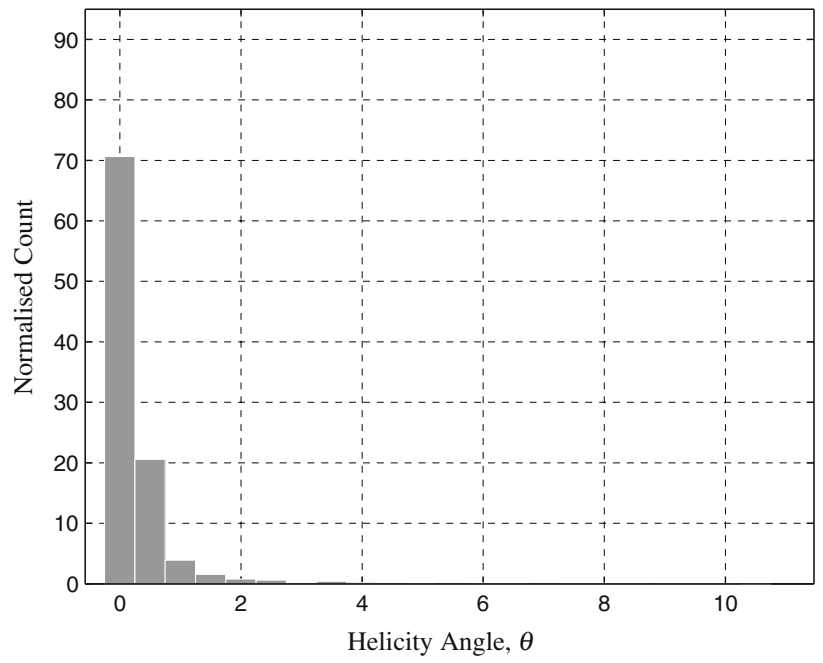

Fig. 23 Histogram showing the frequency of the helical angle computed using a resolution, $d x_{j}=0.025$ for the $\mathrm{ABC}$ flow field that was set up to analyse the effect of resolution on helical coherent structures. At higher resolution, the distribution of helical angles tends to $0^{\circ}$ angle

least dissipation rate, and therefore the maximally helical components in the flow should survive longest as they are the least damped modes.

Mean helical angles of Figs. 14 and 15 show, however, no tendency towards maximal helicity $\left(0^{\circ}\right.$ or $\left.180^{\circ}\right)$ and even with the caveat that the PIV resolution effects illustrated by Figs. 20-23 spreads out the helical angle away from $0^{\circ}$ or $180^{\circ}$, it is clear that the attractive helical angle is actually, $90^{\circ}$, consistent with streamwise vorticity. Thus the conditions expected for the anti-correlation of helicity density and energy dissipation rate anticipated by these 
theories, and actually found in the DNS simulations of Orlandi and Fatica (1997) and Orlandi (1997), are not met for the scales that are resolvable by the dual planar PIV approach used here.

Clearly, there can be multiple reasons as to why structures with mean maximal helicity are not observed in this case. Some points are delineated below:

1. The structures are transient and therefore are not detected by mean turbulence statistics as computed here. To gain a deeper understanding in the evolution of helicity, further experimental investigations need to be carried out, most likely further downstream where residence time has been sufficient for self excitation of helical coherent structures.

2. The structures are smaller in scale than the resolution accuracy of the dual planar PIV technique used here. This could be due to the creation of such structures by instabilities that breakdown turbulent eddies, which may require several stages for significant population of such helical modes.

3. The interrogation planes may be too close to the jet outlet for the residence time for such breakdown stages to have occurred.

4. Due to the the large scale nature of the measurements, there were regions of high shear area as shown in Fig. 6. It is believed that the regions of high shear could affect the dynamics of the flow field causing the vorticity alignments to deviate from isotropic forms significantly as shown previously by Mullin and Dahm (2006b).

5. These helical modes may not be excited due to the statistical symmetry of the (round) jet and the swirling induced in the jet creation, nor by the mechanisms of turbulent instabilities in the flow. In sequels to this work (Regunath (2007)), item 4 is explicitly tested using acoustic actuation of helical modes of instability in jets (kink instabilities).

In this work, we have analysed data using the average of instantaneous quantities and it is intuitive to think that the same results could have been obtained using stereo PIV measurements at different $\mathrm{z}$ positions. However, we are currently working on the next manuscript that clearly shows the difference between averages of the operator applied to the instantaneous quantities (obtained with dualplane PIV) and the operator applied to the average of the quantities (obtained with stereoscopic PIV).

\section{Conclusions}

The described results reports the successful estimation of helicity and energy dissipation terms that were measured quantitatively using the dual-plane dye laser technique. The dual-plane dye laser PIV system was developed and used to analyse helicity in a turbulent swirling flow. The new dual-plane dye laser system consists of a dye laser box that generates two light sheets of different wavelengths using a single laser source. To compute velocity derivatives, three CCD cameras with optical filters are used to capture the separate scattered light from the tracer particles.

The experimental investigations were performed at the $32 \mathrm{~mm}$ distance downstream from the swirl nozzle exit. At this distance, the velocity fields of the tracer particles from the two illuminated light sheets were measured and recorded. From these velocity measurements, all the vorticity components, $\omega_{x}, \omega_{y}$ and $\omega_{z}$ were determined. The average helicity density, $\bar{h}$ for the $32 \mathrm{~mm}$ plane was evaluated using the instantaneous vorticity and velocity components. For the turbulent energy dissipation, the continuity equation was used to infer the $\partial w / \partial z$ velocity gradient and hence with all the velocity gradient components, the turbulent energy dissipation for the $32 \mathrm{~mm}$ plane was evaluated.

From the analysis of results, it was found that for the $32 \mathrm{~mm}$ plane, the regions with maximal helicity corresponded with regions of high energy dissipation. These findings were based on a fluid region where the flow was still in its transitional stage. To fully quantify and understand the evolution of maximal helicity and the relation with energy dissipation, further experimental investigation at various streamwise distances are currently being carried out.

Acknowledgments The authors gratefully acknowledge that this work was supported by the EPSRC Grant (GR/S08695). The authors also wish to acknowledge Oxford Lasers for their help in commissioning the dual-plane dye laser PIV system, as in the assistance of technicians in the University of Sheffield for the construction of the experimental rig. The experimental investigations and procedures that were carried out for this paper comply with the current laws of United Kingdom.

\section{References}

Ganapathisubramani B, Longmire EK, Marusic I, Pothos S (2005) Dual-plane PIV technique to determine the complete velocity gradient tensor in a turbulent boundary layer. Exp Fluids 39(2):222-231

Gupta AK, Lilley DG, Syred N (1984) Swirl flows. Abacus Press, Tunbridge Wells

Hassell DG, Zimmerman WB (2006) Investigation of the convective motion through a staggered herringbone micromixer at low reynolds number flow. Chem Eng Sci 61(9):2977-2985

Hu H, Saga T, Kobayashi T, Taniguchi N, Yasuki M (2001) Dualplane stereoscopic particle image velocimetry: system set-up and its application on a lobed jet mixing flow. Exp Fluids 31(3): 277-293 
Hu H, Saga T, Kobayashi T, Taniguchi N (2002) Simultaneous measurements of all three components of velocity and vorticity vectors in a lobed jet flow by means of dual-plane stereoscopic particle image velocimetry. Phys Fluids 14(7):2128-2138

Kähler CJ (2004) Investigation of the spatio-temporal flow structure in the buffer region of a turbulent boundary layer by means of multiplane stereo PIV. Exp Fluids 36(1):114-130

Kähler CJ, Kompenhans J (1999) Multiple plane stereo PIV: technical realization and fluid-mechanical significance. In: Proceedings of the 3rd international workshop on particle image velocimetry, Santa Barbara, California

Kähler CJ, Kompenhans J (2000) Fundamentals of multiple plane stereo particle image velocimetry. Exp Fluids 29:S70-S77

Kerr RM (1987) Histograms of helicity and strain in numerical turbulence. Phys Rev Lett 59(7):783-786

Kim KC, Yoon SY, Kim SM, Chun HH, Lee I (2006) An orthogonalplane PIV technique for the investigations of three-dimensional vortical structures in a turbulent boundary layer flow. Exp Fluids 40(6):876-883

Kline SJ, McClintock FA (1953) Describing uncertainties in single sample experiments. Mech Eng 75:3-8

Landreth CC, Adrian RJ (1988) Electrooptical image shifting for particle image velocimetry. Appl Opt 27(20):4216-4220

Levich E, Tsinober A (1983a) Helical structures, fractal dimensions and renormalization-group approach in homogeneous turbulence. Phys Lett 96A:292-298

Levich E, Tsinober A (1983b) On the role of helical structures in three-dimensional turbulent flow. Phys Lett A 93(6):293-297

Lourenco L, Krothapalli A (1995) On the accuracy of velocity and vorticity measurements with PIV. Exp Fluids 18(6):421-428

Mathur ML, Maccallum NR (1967) Swirling air jets issuing from vane swirlers. 1. Free jets. J Inst Fuel 40(316):214-225

Moffatt HK (1985) Magnetostatic equilibria and analogous euler flows of arbitrarily complex topology. 1. fundamentals. J Fluid Mech 159(359):378

Moffatt HK, Tsinober A (1992) Helicity in laminar and turbulent flow. Annu Rev Fluid Mech 24:281-312

Mullin JA, Dahm WJA (2005) Dual-plane stereo particle image velocimetry (DSPIV) for measuring velocity gradient fields at intermediate and small scales of turbulent flows. Exp Fluids 38(2): 185-196

Mullin JA, Dahm WJA (2006a) Dual-plane stereo particle image velocimetry measurements of velocity gradient tensor fields in turbulent shear flow. I. Accuracy assessments. Phys Fluids 18(3):035101

Mullin JA, Dahm WJA (2006b) Dual-plane stereo particle image velocimetry measurements of velocity gradient tensor fields in turbulent shear flow. II. Experimental results. Phys Fluids 18(3):035102

Orlandi P (1997) Helicity fluctuations and turbulent energy production in rotating and non-rotating pipes. Phys Fluids 9(7):20452056

Orlandi P, Fatica M (1997) Direct simulations of turbulent flow in a pipe rotating about its axis. J Fluid Mech 343:43-72

Panda J, McLaughlin JK (1994) Experiments on the instabilities of a swirling jet. Phys Fluids 6(1):263-276

Prasad AK (2000) Stereoscopic particle image velocimetry. Exp Fluids 29:103-116

Prasad AK, Jensen K (1995) Scheimpflug stereocamera for particle image velocimetry in liquid flows. Appl Opt 34(30):7092-7099

Regunath GS (2007) Measurements and investigation of helicity in turbulent swirling flow. PhD Thesis

Rogers MM, Moin P (1987) Helicity fluctuations in incompressible turbulent flows. Phys Fluids 30(9):2662-2671

Saikrishnan N, Marusic I, Longmire EK (2006) Assessment of dual plane PIV measurements in wall turbulence using DNS data. Exp Fluids 41(2):265-278

Soloff SM, Adrian RJ, Liu ZC (1997) Distortion compensation for generalized stereoscopic particle image velocimetry. Meas Sci Technol 8:1441-1454

Tsinober A, Levich E (1983) On the helical nature of 3-dimensional coherent structures in turbulent flows. Phys Lett A 99(6-7):321-324

Wallace JM, Balint JL, Ong L (1992) An experimental-study of helicity density in turbulent flows. Phys Fluids A Fluid Dyn 4(9):2013-2026

Zimmerman WB (1996) Fluctuations in passive scalar concentration convected by a helical flow in a conducting fluid. Phys Fluids $8(6): 1631-1642$ 